\title{
- Physics programme in fixed-target mode with the LHCb experiment at CERN
}

\section{Lucio Anderlini*广}

Istituto Nazionale di Fisica Nucleare - Sezione di Firenze, Sesto Fiorentino, Italy

E-mail: Lucio.Anderlini@fi.infn.it

The LHCb experiment, designed for $b$-physics, introduced SMOG (System for Monitoring the Overlap with Gas) in 2015 to improve the luminosity measurements studying the beam profile through the collisions of the LHC protons with gas atoms injected in the beam-pipe. Protongas collisions are used today to study production cross-sections in fixed-target mode, exploring a kinematic region otherwise unaccessible. In this contribution, I review the programme and present recent measurements on the production of heavy flavours in $p$-Ar collisions and production of antiprotons in $p-\mathrm{He}$ collisions. The latter will contribute to reduce the dominant contribution to the uncertainty on the theoretical predictions of the antiproton flux in primary cosmic rays, an excess of which would represent evidence for New Physics.

EPS-HEP 2017, European Physical Society conference on High Energy Physics 5-12 July 2017

Venice, Italy

* Speaker.

${ }^{\dagger}$ on behalf of the LHCb Collaboration 
The LHCb experiment is a forward single-arm spectrometer designed to study the heavy hadrons produced in $p p$ collisions at the LHC [1]. Today, given the notable extension of the physics programme spanning from jet reconstruction to the study of heavy-ion collisions, it is considered a truly general-purpose experiment in the forward region.

The design of the detector includes a Vertex Locator (VELO) aiming at an excellent discrimination between tracks originating directly from the primary collisions, called prompt, and tracks originating from the decay of a weakly decaying particle; a reduced material budget before the calorimeters improves the momentum and mass resolution; and outstanding particle identification performance are obtained combining the response of two RICH detectors, a Calorimeter System and five Muon stations separated by iron walls.

In 2014, in order to improve the measurement of the integrated luminosity, whose uncertainty enters as dominant systematic in all the production measurements, a technique complementary to the "Van Der Meer scan" was developed. The new method, named "beam-gas imaging", was based on the study of the collisions of the beam protons with a noble gas injected in the beam-pipe through a device named SMOG (System for Measuring the Overlap with Gas), achieving the world's best measurement of the luminosity at a bunched collider with a total uncertainty of $1.16 \%$ [2].

It became evident rather quickly that the opportunity of studying the physics of the proton-gas collisions at the energies made available at the LHC is rather unique. In this write-up, I discuss two physics cases for the proton-gas collisions: the study of cold nuclear matter effects through the production measurements of hidden- and open-charm states in $p$-Ar collisions at $\sqrt{s_{N N}}=110$ $\mathrm{GeV}$; and the measurement of the production cross-section of antiprotons in $p-\mathrm{He}$ collisions, emulating the conditions of primary cosmic rays interacting with the interstellar medium, composed of Hydrogen and Helium.

\section{Heavy Flavour Production in $p-$ Ar collisions}

The study of Heavy Flavour production in heavy ion collisions is considered as one of the most effective probes of collective behaviour while it should be affected by the hot and dense regime of Quark-Gluon Plasma (QGP), through the phenomena known as $c \bar{c}$ suppression and recombination.

It is believed that QGP is not formed in proton-induced reactions of nuclear targets, studying quarkonium production in proton-ion collisions is therefore a robust baseline which enables to identify phenomena related to QGP in ion-ion collisions.

The LHCb Collaboration has recently reported the first analysis of heavy flavour production in fixed-target mode at the LHC [3]. The decays $J / \psi \rightarrow \mu^{+} \mu^{-}$and $D^{0} \rightarrow K^{-} \pi^{+}$are studied to measure the production of $J / \psi$ and $D^{0}$ mesons in $p-A r$ collisions at $\sqrt{s_{N N}}=110 \mathrm{GeV}$. The dataset was collected in a dedicated Run lasted seventeen hours, in 2015.

Figure 1 reports the invariant mass distributions of the $K^{-} \pi^{+}$and $\mu^{+} \mu^{-}$combinations displaying evident contribution from $D^{0}$ and $J / \psi$ decays respectively. The number of $D^{0}$ decays obtained with an unbinned extended maximum likelihood fit to the dataset is $6451 \pm 90$, while $482 \pm 23$ signal candidates are found for $J / \psi$.

While the measurement of the integrated luminosity of the data sample is pending, it is already possible to compare the theoretical expectations to the efficiency-corrected yields as functions of the rapidity and transverse momentum of the decaying hadron. Figure 2 shows good agreement 

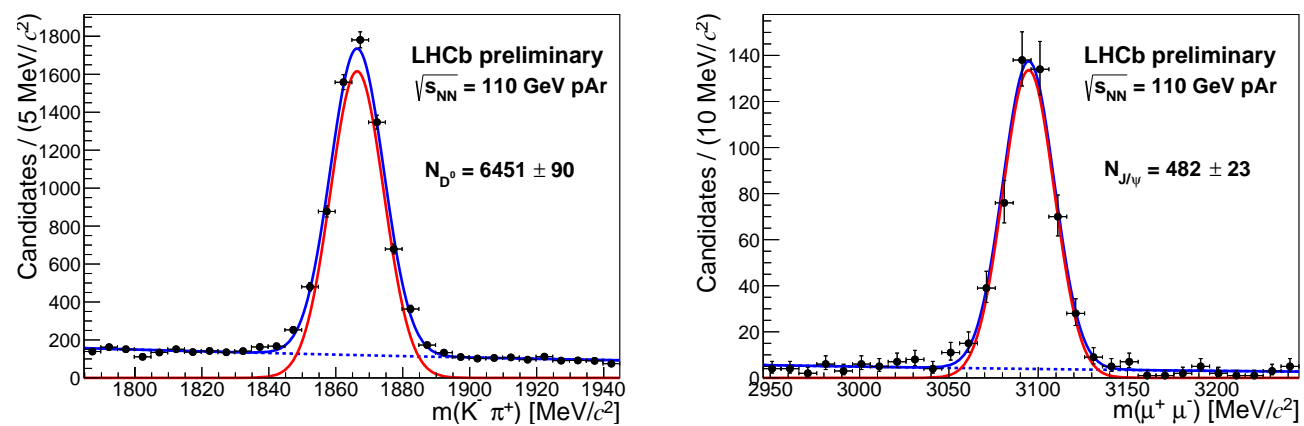

Figure 1: Mass distributions of $K^{-} \pi^{+}$at left and $\mu^{+} \mu^{-}$at right displaying evident contribution from $D^{0}$ and $J / \psi$ decays, respectively. The Figure was made public in Ref. [3].
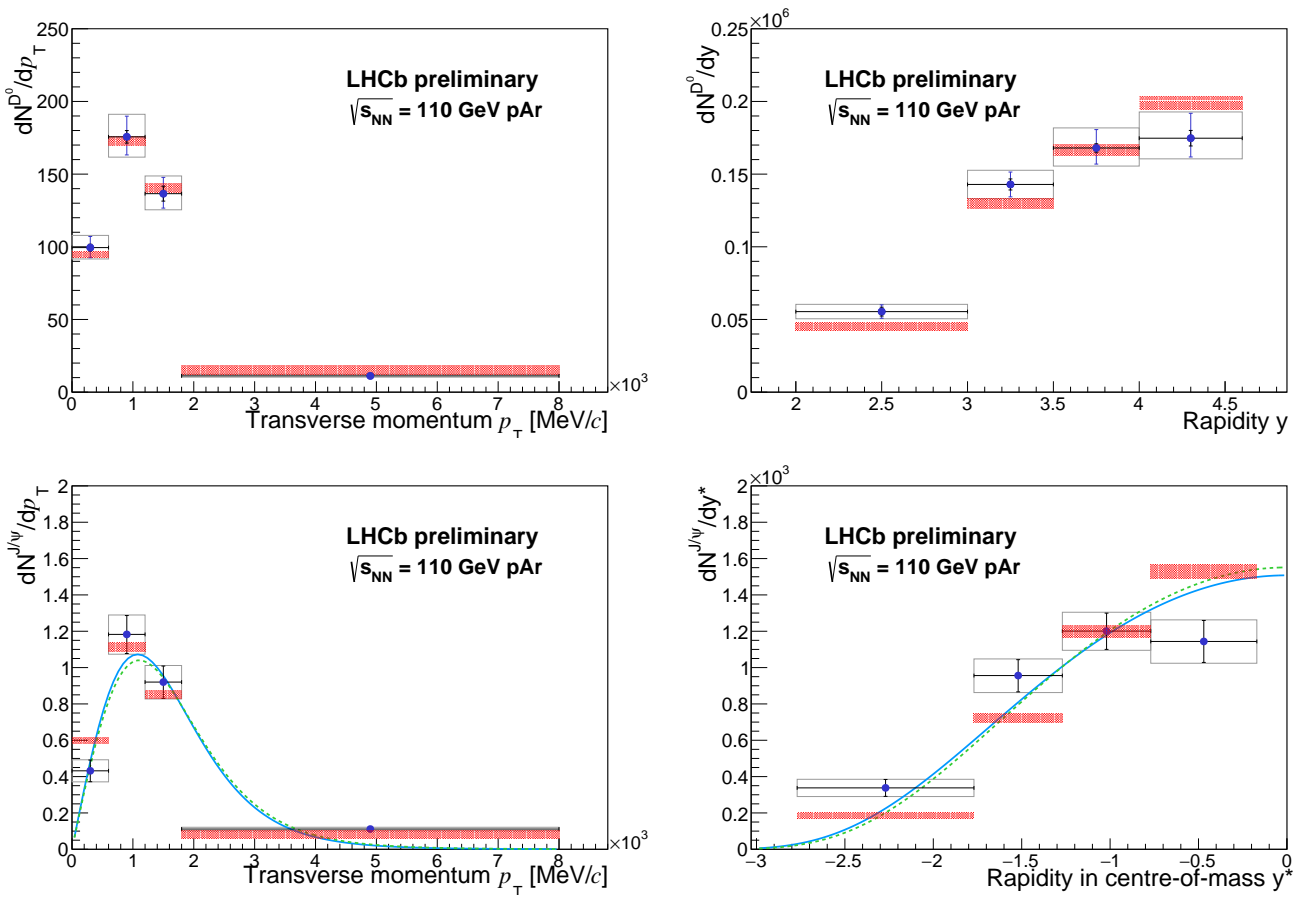

Figure 2: Transverse momentum (left) and rapidity (right) spectra of the $D^{0}$ (top) and $J / \psi$ (bottom) mesons as reconstructed from $p$-Ar collisions at $\sqrt{s_{N N}}=110 \mathrm{GeV}$. The experimental data, shown as marker with error bars (statistical uncertainties) and boxes (total uncertainties), are superposed to the predictions of Pythia [5] and, for the $J / \psi$ only, to the phenomenological parametrizations described in Ref. [6].

of the experimental data with the predictions obtained with Pythia [5] and the phenomenological parametrization described in Ref. [6].

\section{Antiprotons production in $p-\mathrm{He}$ collisions}

In the theory community there is a consensus on the fact that if the dark matter can decay or annihilate to ordinary matter, the flux in cosmic-rays of antiparticles should be significantly enhanced [7]. The attention on such predictions increased already in 2009 when the PAMELA ex- 


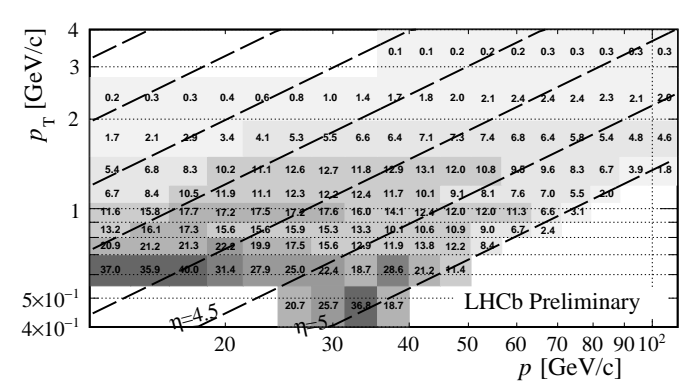

Figure 3: On top, number of antiprotons in $10^{3}$ units. At right, the double differential production cross-section of antiprotons compared to the predictions of EPOS. Both figures are obtained analysing the dataset of $p$-He collisions with $\sqrt{s_{N N}}=110 \mathrm{GeV}$ described in the text. Figures were made public in [4].

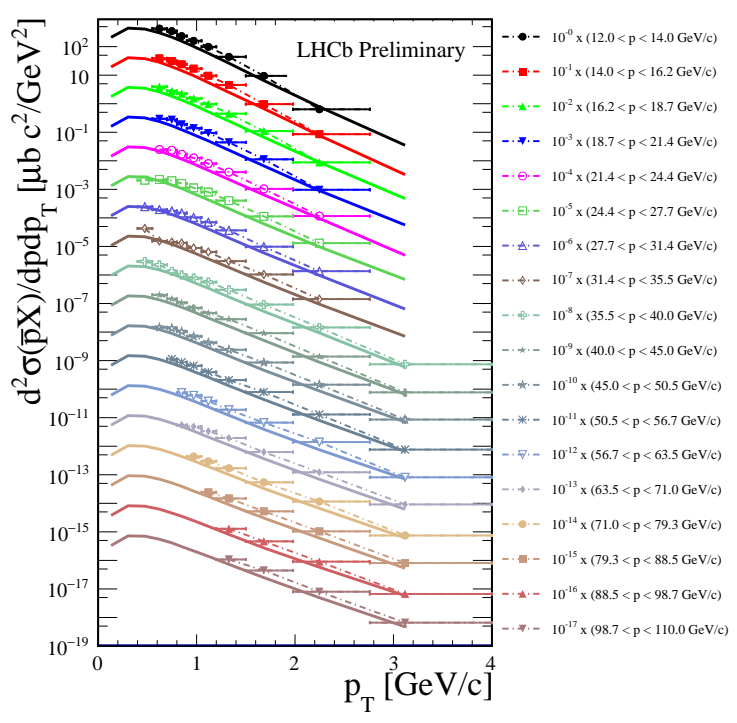

periment reported a dependency of the antiproton flux on their kinetic energy milder than predicted [8]. The result was then confirmed in 2016 by the AMS-02 experiment, with improved precision and statistics [9].

A careful reconsideration of the theoretical uncertainties, however, shows that the dominant source of the systematic error is due to the poor knowledge of the probability of producing antiprotons in the collisions between the primary cosmic protons and the interstellar medium, dominated by Hydrogen and Helium. The contribution to the cosmic antiproton flux from these secondary production mechanism is estimated extrapolating the production cross-section of antiprotons in proton-proton and proton-carbon collisions at a much lower energy [10], since no direct measurement was performed at the energy scale of $\sqrt{s_{N N}} \sim 100 \mathrm{GeV}$.

The LHCb experiment, with the SMOG extension for fixed-target mode, is perfectly suited to achieve the first measurement of the antiproton production cross-section in $p-\mathrm{He}$ collisions [4]. The energy of the proton in the eighteen-hour run studied for the first analysis was $6.5 \mathrm{TeV}$, corresponding to an energy in the center of mass $\sqrt{s_{N N}}=110 \mathrm{GeV}$. Neglecting the per-mill contribution of leptons, the negative-charge particles produced in the $p-\mathrm{He}$ collision are classified into pions, kaons and antiprotons. The yield of each category is measured in kinematic bins through a binned likelihood fit to the combined response of the two RICH detectors. The uncertainty on the yields obtained is dominated by the systematic uncertainty on the quality of the template distributions for pions, kaons and protons, but it is controlled below 10\% in most bins, and below $30 \%$ also in the kinematic bins at the boundary of the acceptance. The antiproton yield obtained from the fit is then corrected for the reconstruction and selection efficiency as obtained from simulation.

Since the gas density is not known to a better accuracy than the $10 \%$ achieved for the uncertainty on the yields, a normalization channel is used to convert the yields into a cross-section. The channel chosen as normalization is the elastic scattering of the beam protons on the atomic electrons. The cross-section of the elastic scattering is purely electromagnetic and it is therefore theoretically known with excellent precision. Experimentally, elastic scattering events are easily identified since a single negative track consistent with an electron is observed in the detector 
acceptance. The background due to photon conversion and pions pairs produced in soft QCD interactions are charge symmetric and can be statistically subtracted studying the distributions of the positive-charge single-track events.

The results, shown in Figure 3, are compared to the predictions of EPOS LHC [11]. The momentum spectrum is well predicted, while the absolute production rate in data is larger by a factor $\sim 1.5$.

To study the contribution to the cross-section of events with multiple antiprotons, the total inelastic cross-section was also measured and compared to EPOS predictions,

$$
\sigma_{\text {inel }}^{\mathrm{LHCb}}=(140 \pm 10) \mathrm{mb} \quad \sigma_{\text {inel }}^{\mathrm{EPOS}}=118 \mathrm{mb} \quad \frac{\sigma_{\text {inel }}^{\mathrm{LHCb}}}{\sigma_{\text {inel }}^{\mathrm{EPOS}}}=1.19 \pm 0.08 .
$$

The result implies a significantly larger antiproton multiplicity per inelastic collision with respect to EPOS LHC.

\section{Conclusion and outlook}

The LHCb experiment has demonstrated to excel in fields beyond those foreseen in the original design. The fixed-target mode, obtained with SMOG, opens the experimental programme of the collaboration to a new energy range which is intermediate between the fixed-target collisions at the SPS and the collider-mode of the LHC. The highly-boosted center-of-mass system allows to access a wide region of the Feynman $x_{F}$, including large negative (backward) $x_{F}$ and very small forward $x_{F}$, since rapidity is shifted by -4.7 .

The LHCb experiment has already collected abundant samples of $p-\mathrm{He}, p-\mathrm{Ne}, p-\mathrm{Ar}$ samples at $\sqrt{s_{N N}}=87$ and $110 \mathrm{GeV}$, whose analysis is currently ongoing. An upgraded SMOG version will allow a better control of the gas density in order to add external constraints to the measurement of the luminosity in fixed-target mode, which will complement or supersede the technique based on the elastic proton-electron scattering.

\section{References}

[1] The LHCb Collaboration, JINST 3 (2008) S08005, Int. J. Mod. Phys. A 30 (2015) 1530022

[2] The LHCb Collaboration, JINST 9 (2014) P12005

[3] LHCb Conference Report, LHCb-CONF-2017-001, cds.cern.ch/record/2255650

[4] LHCb Conference Report, LHCb-CONF-2017-002, cds.cern.ch/record/2260835

[5] T. Sjöstrand et al., JHEP 05 (2006) 026

[6] F. Arleo et al., JHEP 05 (2013) 155

[7] F. Donato et al., Phys. Rev. D62 043003

[8] The PAMELA Collaboration, Nature 458 (2009) 607-609

[9] The AMS Collaboration, Phys. Rev. Lett. 117 (2016) 091103

[10] The NA49 Collaboration, Eur. Phys. J. C65 (2010) 9-63; Eur. Phys. J. C73 (2013) no.4, 2364

[11] T. Pierog et al., Phys. Rev. C92 (2015) 034906 